\title{
THE SHAPES AND SHAPING OF PLANETARY NEBULAE
}

\author{
Bruce Balick ${ }^{1}$ \\ University of Washington \\ Dept. of Astronomy, FM-20 \\ Seattle, Washington 98195 \\ U.S.A.
}

\begin{abstract}
One of the most striking attributes of planetary nebulae are their complex, yet highly symmetric shapes. The process(es) which shape planetaries are only beginning to be understood. It is proposed that the morphologies of most PNs can be understood within the context of the "interacting winds" wherein a fast but light wind driven by the nucleus rams into an older, slower, and more massive wind, or red giant envelope ("RGE") ejected earlier.

In order to explain the shapes of noncircular PNs, it seems necessary to hypothesize that the slow wind was originally ejected with an enhanced density along an equatorial plane. The morphologies of nearly all PNs can be understood through two basic parameters: the equatorial density contrast in their RGEs, and the degree of interaction between the fast and slow winds.
\end{abstract}

\section{INTRODUCTION}

Winds are now recognized as important in the shaping of many types of nebulae (e.g. Pikel'ner 1968, 1973; Kwok et al. 1978; Kwok 1980, 1982; Kahn 1983, Okorokov et al. 1985; Volk and Kwok 1985; Kahn and West 1985). Kwok and his collaborators have been instrumental in developing the "interacting winds" model for planetary nebulae (hereafter PNs) wherein fast, light winds from a central star interact hydrodynamically with the former outer layers of the star ejected as the star evolved from the red-giant stage. The "fast wind" is characterized by mass flows of $\sim 10^{-7}$ to $\sim 10^{-8}$ at radial velocities of about $10^{3} \mathrm{~km} \mathrm{~s}^{-1}$.

According to one-dimensional model calculations, the hydrodynamic interaction generates two stable shocks. The outermost of the two is the region where a hot, relatively high pressure interior bubble energized by the winds interfaces to the as-yet unaffected RGE. In times short compared to the dynamical age of the RGE the outer shock becomes associated with a snowplow, which is observable as a dense and bright "rim" between the hot interior bubble and the RGE. The snowplow is sustained by the negative radial pressure gradient caused by the wind. The second

1 Visiting Astronomer, Kitt Peak National Observatory, National Optical Astronomy Observatories which is operated by the Association of Universities for Research in Astronomy, Inc. under contract with the National Science Foundation. 
shock is reverse shock which settles into place near the star at the points where the pressure of the radially streaming wind is matched by the pressure of the hot bubble upstream.

The simple models are in qualitative accord with the morphologies of the relatively rare circular PNs such as NGC 1535, NGC 6894, and IC 3568. Even for simple (i.e. round) PNs, detailed agreement between observation and theory must await knowledge of the changes in the properties of the fast wind as the PN nucleus evolves, and such knowledge is elusive (see Kwok 1987 and Schoenberner 1987 for discussions).

However, the typical PN is not round. Clearly either the RGE or the fast winds (or both) are nonaxisymmetric. Can the precepts of the interacting wind model be applied to explain the shapes of non-round PNs? This is the underlying scientific question which motivates the work described here.

The scientific question is a large one, and one which requires computation of full two- or three-dimensional time-dependent hydrodynamic model calculations. Such calculations are difficult and unavailable, and they rely on a knowledge of initial and boundary conditions (i.e. gas density and motion distributions) which are yet to be supplied by observers. Hence, the question is not yet answerable.

\section{THE DATA BASE}

Observations reported here were made with the 2.1-m telescope of the Kitt Peak National Observatory through a variety of narrow ( $\sim 10-20 \AA$ bandpass) emission line filters. The detector was a Texas Instruments CCD whose large dynamic range makes possible the study of the interrelations of both subtle background features (such as the RGE) and bright highlights (e.g. the bright rim) in a single deep exposure. The data are described and presented by Balick $(1987 \mathrm{a}, \mathrm{b})$. Selected images shall be presented later.

\section{MORPHOLOGICAL SEQUENCE}

Based on these data, Balick (1987b) proposed a morphological sequence which is illustrated in fig. 1. All arrows in the figure should be ignored for the present. From left to right there are classes of PNs which are based on the symmetry of the of bright parts of the nebula. The simplest $P N s$ are round $(R)$ and are shown in the left column. PNs in the center column are designated as "ellipticals" (E), and those on the right as "butterflies" (B). In each column, from top to bottom, PNs are designated by types, i.e. "early" (e), "middle" (m), and "late" (l). PNs can be designated as eE (early elliptical), mB (middle butterfly), etc. Obviously, there will be PNs of intermediate classes and types.

In order to be useful, a morphological sequence must encompass a major fraction of known objects. Balick (1987b) argues that most, though not all, PNs are accommodated by the sequence in fig. 1. Of Balick's $51 \mathrm{PNs}, 29$ of the best examples which fit into the classification scheme are shown in figs. 2, 3, and 4 . Another eleven apparently fit into the sequence but are sufficiently small in angular size or faint that they are not shown here.

Another eleven of Balick's 51 PNs fail to fit into the sequence. "Lumpy" PNs such as NGC 2452, NGC 6210, IC 4593, and J 320 seem to consist of many knots, 


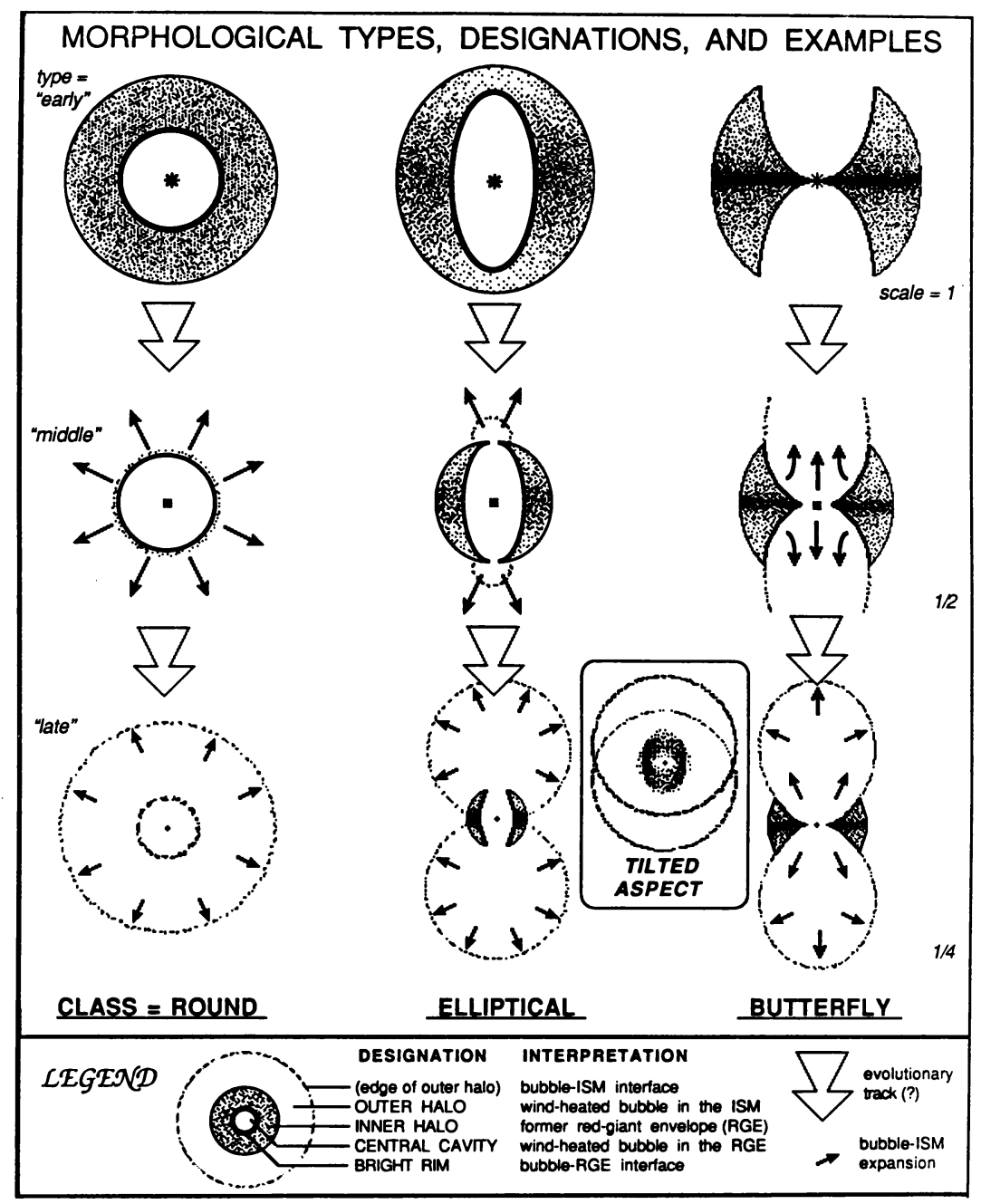

Fig. 1 - Schematic illustrations of two-dimensional cuts through the centers of various morphological archetypes of PNs. Grey regions are remaining regions of the RGE (inner halos), where grey tone is related to gas density. Heavy lines indicate regions where the stellar wind has pushed portions of the RGE into snowplows (rims). The morphological types are defined by the shapes (after projection onto the sky) of inner halos and rims.

Light, broken lines indicate the leading edge of the interface between winds and the interstellar medium which can be identified with outer halos. Wide, white arrows indicate evolutionary pathways through the diagram, and thin black arrows indicate the directions of the advancing wind-ISM interface. Scale changes among the figures are indicated by the size of the icon representing the central star. 


\begin{tabular}{rc}
\multicolumn{2}{c}{ ROUND PNS } \\
EARLY TYPE & scale (pC) \\
IC 418 & 0.08 \\
BD+30 3639 & 0.10 \\
IC 3568 & 0.24 \\
NGC 1535 & 0.55
\end{tabular}
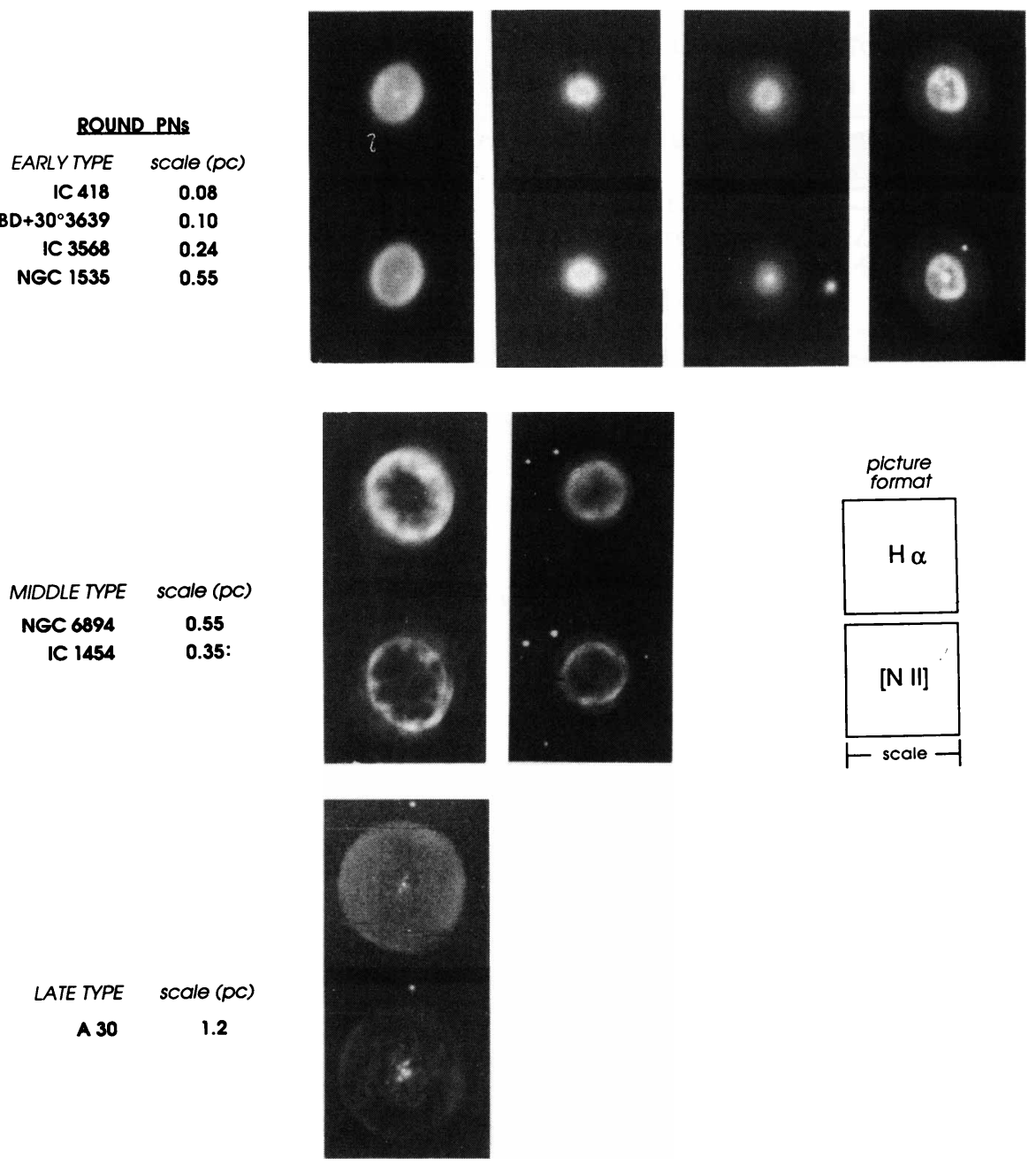

Fig. 2 - Examples of Round PNs of various types. The pictures are from CCD images through $\mathrm{H} \alpha$ (top panels) and [N II] (bottom panels). The contrast has been altered to emphasize faint features while not suppressing highlights in the images. 


\begin{tabular}{lc}
\multicolumn{2}{c}{ ELLIPIICAL PNs } \\
EARLY TYPE & scale (PC) \\
NGC 3242 & 0.28 \\
NGC 6826 & 0.56 \\
NGC 7662 & 0.13 \\
NGC 2022 & 0.44
\end{tabular}

EARLY-MIDDLE scale (pC)

$\begin{array}{rr}\text { NGC 2610 } & 0.78 \\ \text { NGC 7009 } & 0.22 \\ \text { NGC 7354 } & 0.65 \\ \text { IC 289 } & 0.4:\end{array}$

$\begin{array}{cc}\text { MIDOLE TYPE } & \text { scale (pC) } \\ \text { NGC 40 } & 0.15 \\ \text { NGC 7048 } & 0.43 \\ \text { NGC 7139 } & 0.74\end{array}$
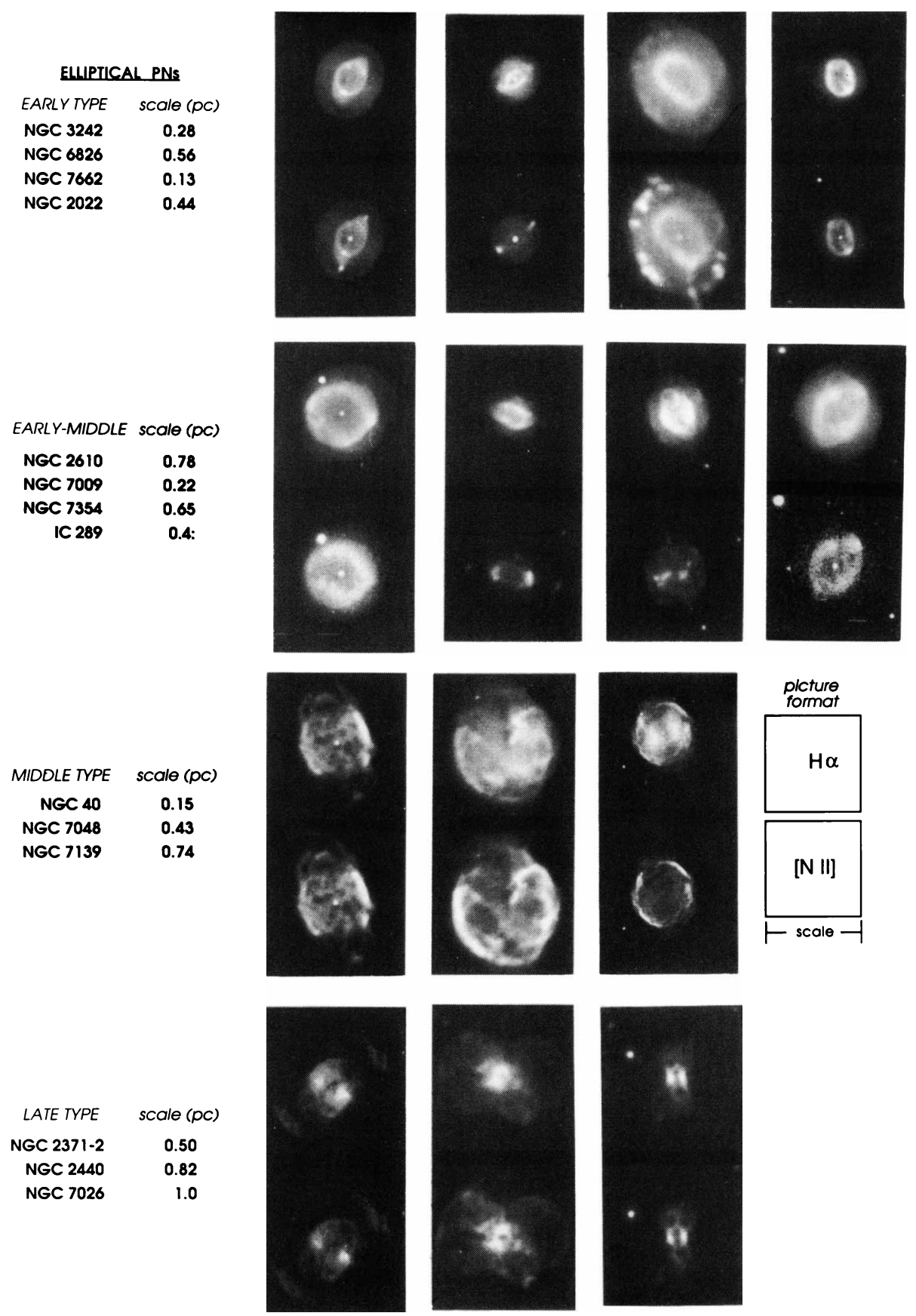

Fig. 3 - Examples of Elliptical PNs of various types. 


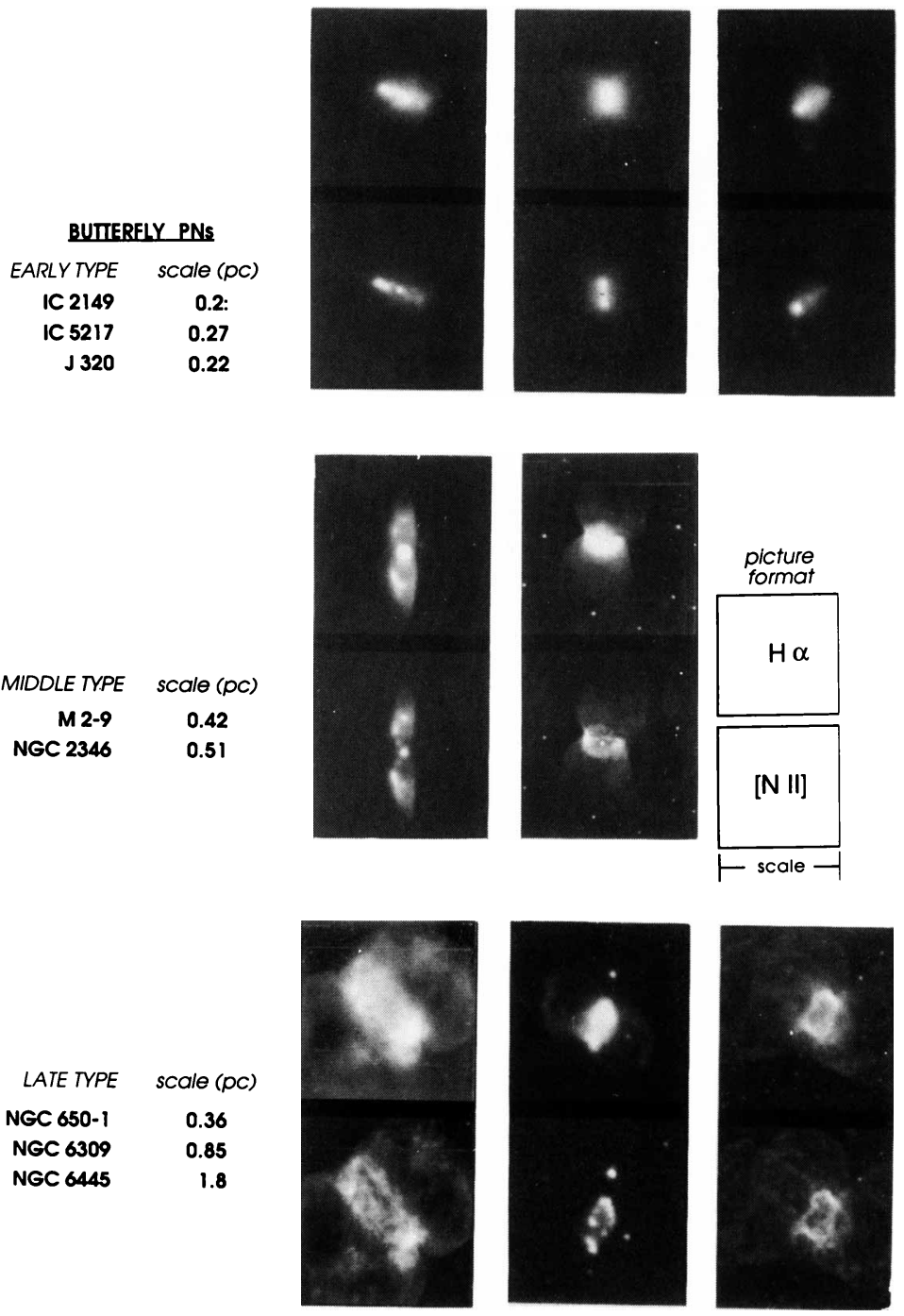

Fig. 4 - Examples of Butterfly PNs of various types. 
often in pairs on opposite sides of the central star. NGC 1514 and NGC 3587 are "amorphous" - they contain none of the bright fine structure which a critical defining characteristic of the present sequence.

NGC 6543, NGC 7008, and NGC 7293 are called "peculiar". Even though peculiar PNs are extremely symmetric, their morphologies are apparently singular among all PNs. Finally, NGC 7027 is an example of an anomalous PN whose intrinsic structure (as observed at IR and radio wavelengths) fits into the morphological scheme, but whose optical appearance is heavily affected by strong and patchy foreground extinction. Finally, it must be noted that PNs are classified according to their projected appearance on the sky. Some PNs may be misclassified as a result. NGC 6720, NGC 6781, and IC 1747 are likely improperly classified candidates owing to projection effects.

\section{PHYSICAL SIGNIFICANCE}

The goal for defining a morphological sequence, whether in biology or astronomy, is to attempt to provide an organizational framework within which the patterns of morphologies might find an interpretation. The immediate goal here is to determine whether the shapes of $\mathrm{PNs}$ are consistent with a hydrodynamical model of interacting winds. Although consistency is not to be confused with proof, the establishment of consistency between theoretical expectations and observations is a very important motivation for possible future research.

Since no detailed multi-dimensional hydrodynamical models based on realistic initial and boundary conditions are available, it is important to emphasize that the attempt to show consistency between data and theory is based on theoretical expectations, not calculations. Such an approach is treacherous, and in the long term the proposed morphological sequence might be found to have little relevance to the manner in which PNs evolve!

\subsection{The Observed Environments}

As seen in nearly all of the early-type PNs of figs. 2 - 4, the slow wind is taken to have a higher density, and thus higher pressure, along an equatorial axis that along the symmetry axis of the equator (i.e. along the polar axis). The equatorial-polar density ratio, or density contrast, is unity for circular PNs and increases regularly for elliptical and butterfly PNs. The equatorial density enhancement is particularly obvious in pictures of e-m E and B PNs such as NGC 650-1, NGC 2346, NGC 2610, NGC 3242, NGC 6826, NGC 7354, and NGC 7662.

The equatorial zones of butterfly nebulae may be sufficiently dense to be optically thick to ionizing radiation. Such RGEs might be described as dense disks, portions of which are largely neutral. IR and radio molecular studies are showing the existence of these disks (e.g. Zuckerman and Gatley 1988; Bieging and NguyenQuang-Rieu, preprint; and Balick, Gatley, and Zuckerman, private communication).

The process that ejects the RGE with is not clearly understood. Poe and Friend (1986), Friend and Abbott 1986 and their coworkers have argued that the massive stars are likely to contain similar bands. 


\subsection{Early Evolution}

In the subsequent discussions we adopt the general conditions of the interacting winds model (e.g. Kwok 1982 and Volk and Kwok 1985). The RGEs expand homologously until overtaken by the fast winds. We assume that the fast wind is isotropic as it leaves the stellar photosphere.

Round PNs show no evidence of density variations other than radial. Thus one expects the one-dimensional models to describe the nebular structure. For such PNs the snowplow associated with the outer shock can be seen as a circular bright rim along the inner edge of the RGE. NGC 1535, IC 3568 (fig. 1) and Shapley 1 (Pottasch 1984, p. 3) are archetypical eR PNs. Inside the bright rim is a hot bubble which is too sparse and highly ionized to be detected at visible wavelengths. Ultraviolet absorption line studies (e.g. Kaler et al.1987) confirm that there exists a transition region between the nebula and the hotter, more highly ionized interior of some PNs.

In elliptical and butterfly PNs the outer shock pushes more quickly towards the low-density polar regions than the equator. In eE-type PNs the snowplow is prolate ellipsoidal or even peanut-shaped, and is seen as a closed bright rim when projected on the sky. Simplified models of such nebulae have been computed by Kahn and West (1985). Balick et al.(1987) have investigated how eE PNs produce the collimated gas flows seen frequently in this class of PNs. Their results are summarized elsewhere in this volume (Balick et al.1988).

\subsection{Subsequent Development}

If the stellar wind continues to maintain a higher pressure in the bubble interior than in the RGE, the outer shock will drive outward. Eventually the outer shock will reach the edge of the $R G E$ and, if the density near the RGE edge decreases, instabilities may develop (see, e.g., NGC 40, NGC 6894, NGC 7048, and the halo of NGC 6543 in Millikan 1974). Most PNs in this evolutionary state are of the middle type, and are drawn along the center row of fig. 1. mE PNs are characterized by a pair of polar protruberances, as seen in fig. 3. Other examples of $\mathrm{mE}$ PNs might be Abell 43 and 72 (see Pottasch 1984, pp 12 and 13) and NGC 6905.

In cases where the density contrast of the RGE was originally large, the development of rapidly expanding bipolar lobes is likely to begin very quickly. Models of the development of bipolar lobes in disk-dominated environments have been developed by Icke and Choe (1987), Icke et al.(1988), and Pudritz and Norman (1986), among others. The dynamics of such systems is particularly easy to study in PNs of the butterfly class such as NGC 2346 and M 2-9 owing to their relatively high surface brightness and low extinction.

Once pierced, the RGE can no longer contain the bubble. The hot material expands nearly adiabatically until eventually confined by the ISM or material ejected earlier in the lifetime of the star. Except for round PNs, two large lobes will develop. These lobes will be limb brightened as the shocks along their leading edge again form snowplows in the ambient medium. Often the lobes will be faint and very difficult to detect (Chu, Jacoby, and Arendt, 1987; Jewitt, Danielson, and Kupferman 1986). Their expansion motions are likely to be highly supersonic. Heathcote and Weller (1987) and Balick et al.(1988) report that the two outer lobes of NGC 2440 are expanding at $\sim 75 \mathrm{~km} \mathrm{~s}^{-1}$. Other late-type PNs which might be in a similar stage of evolution are shown in the bottom rows of figs. 3 and 4. 


\section{CONCLUSIONS}

If one adopts the hypothesis that PNs are shaped by fast and isotropic stellar winds which interact with a red giant envelope expelled earlier with some degree of equatorial density enhancement, then a sequence of evolutionary states for PNs can be predicted. The shapes of most PNs fit nicely into the expected morphological categories (with some exceptions, of course). While such an exercise does not prove that PNs are shaped by winds, the morphological agreement between expectation and reality justifies further research.

There are several important research directions that are required before the process of wind-shaping is ready for serious credibility. On the theoretical side, two-dimensional hydrodynamic model calculations with both photon and collisional heating must be developed. It is up to the observers to determine appropriate initial and boundary conditions for these calculations. Among the missing data are the mass and velocity distributions of RGEs in PNs and their evolutionary predecessors, and the kinematics of the gas in the rims, RGEs, and other important (and readily observable) nebular structural components. Such studies are underway; however, the enormity of the task invites the participation of everyone.

\section{ACKNOWLEDGEMENTS}

It is a pleasure to thank many cooperative people who have been working to understand the structure of PNs. My collaborations with Heather Preston and Vincent Icke have been especially rewarding, both personally and scientifically. George Jacoby and You-Hua Chu have enthusiastically shared their latest results with us on many occasions. Important discussions and encouragement has been freely offered by Lawrence Aller, Ian Gatley, Sun Kwok, Stuart Pottasch, Yervant Terzian, and Ben Zuckerman, to name only a few. Financial support was received through grants AST 82-08041, 83-10552 (for astronomical image processing at the University of Washington), and 86-12228 from the National Science Foundation.

\section{REFERENCES}

Balick, B. (1987a). Sky and Tel. 73, 125.

Balick, B. (1987b). Astron. J. 94, 671.

Balick, B., Preston, H.L., and Icke, V. (1987). Astron. J. (December 1987 issue).

Balick, B., Preston, H.L., and Icke, V. (1988). This volume.

Bieging, J.H. and Nguyen-Quang-Rieu (1987). Preprint.

Chu, Y.-H., Jacoby, G.H., and Arendt, R. (1987). Preprint.

Friend, D.B., and Abbott, D.C. (1986). Astrophys J. 311, 701.

Icke, V., and Choe, S.-U. (1987). Submitted to Astron. Astrophys.

Icke, V., Preston, H.L., and Balick, B. (1988). In preparation.

Jewitt, D.C., Danielson, G.E., and Kupferman, P.N. (1986). Astrophys. J. 302, 727.

Kahn, F.D. (1983). In Planetary Nebulae, IAU Symposium No. 103, edited by D. R. Flower (Reidel, Dordrecht), p. 305.

Kahn, F.D., and West, K.A. (1985). Mon. Not. R. Astron. Soc. 212, 837.

Kaler, J.B. Feibelman, W.A., and Henrichs H.F. (1987). Preprint. 
Kwok, S. (1980). J. R. Astron. Soc. Canada 74, 216.

Kwok, S. (1982). Astrophys. J. 258, 280.

Kwok, S. (1987). In Late Stages of Stellar Evolution, edited by S. Kwok and S.R. Pottasch (Reidel, Dordrecht), p. 321.

Kwok, S., Purton, C.R., and Fitzgerald, M.P. (1978). Astrophys. J. Lett. 219, L125.

Millikan, A.G. (1974). Astron. J. 79, 1259.

Okorokov, V.A., Shustov, B.M., Tutukov, A.V., and Yorke, H.W. (1985). Astron. Astrophys. 142, 441.

Pikel'ner, S.B. (1968). Astrophys. Lett. 2, 97.

Pikel'ner, S.B. (1973). Astrophys. Lett. 15, 91.

Poe, C.H., and Friend, D.B. (1986). Astrophys. J. 311, 317.

Pottasch, S.R. (1984). Planetary Nebulae (Reidel, Dordrecht).

Pudritz, R.E., and Norman, C.A. (1986). Can. J. Phys. 64, 501.

Schoenberner D. (1986). In Late Stages of Stellar Evolution, edited by S. Kwok and S.R. Pottasch (Reidel, Dordrecht), p. 337.

Volk, K.M. and Kwok, S. (1985). Astron. Astrophys. 153, 79.

Weller, W.G. and Heathcote, S.R. (1987). In Late Stages of Stellar Evolution, edited by S. Kwok and S.R. Pottasch (Reidel, Dordrecht), p. 409.

Zuckerman, B. and Gatley, I. Astrophys. J. (Jan. 1988 issue). 